\title{
Editorial: Recent Advances in Service Life Prediction of Bridges and Structures
}

\author{
Marija Kušter Marić ${ }^{1 *}$ and Emilio Bastidas-Arteaga ${ }^{2,3}$ \\ ${ }^{1}$ Faculty of Civil Engineering, University of Zagreb, Zagreb, Croatia, ${ }^{2}$ Research Institute in Civil and Mechanical Engineering UMR \\ CNRS 6183, Université de Nantes, Nantes, France, ${ }^{3}$ Laboratoire des Sciences de l'Ingénieur pour l'Environnement (LaSIE UMR \\ CNRS 7356), La Rochelle Université, La Rochelle, France
}

Keywords: numerical model, degradation mechanism, durability, service life, climate change, measurements

Editorial on the Research Topic

Recent Advances in Service Life Prediction of Bridges and Structures

The global issue of premature deterioration in bridges and other structures requires a switch from prescriptive to performance-based durability design of infrastructure, taking into account specific environmental exposure and a combination of actions to which a structure will be exposed during its service life.

Although many numerical models for service life prediction have been developed in the last four decades, their application on real structures is still not on a satisfactory level. This is due to the complexity of mechanical, physical, thermal and chemical processes related to the deterioration processes, but also due to difficulty in quantifying the material, structural, environmental and climate performance indicators and their interactions. In addition, there are several sources of uncertainty and spatial variability related to deterioration processes, exposure, models, inspection, etc. These uncertainties and spatial variability affect service life predictions and should be quantified and considered in a comprehensive form.

This Research Topic deals with the new advances reached in the last years in the field of service life prediction (deterministic or probabilistic) of bridges and different type of infrastructure, including the following topics:

Specialty section: This article was submitted to Computational Methods in Structural Engineering,

a section of the journal Frontiers in Built Environment

Received: 17 November 2020 Accepted: 08 December 2020 Published: 15 January 2021

Citation:

Kušter Marić $M$ and Bastidas-Arteaga E (2021) Editorial: Recent Advances in Service Life Prediction of Bridges and Structures. Front. Built Environ. 6:630554. doi: 10.3389/fbuil.2020.630554

- Service life prediction models for determining the time to repair

- Life cycle and service life assessment models of concrete, steel and wooden structures

- Impact of hurricane hazard and climate change on service life prediction

- Case studies

- New methods for structure durability testing in laboratory and on site

- Durability of ultra-high-performance concrete structures

- Service life design in the framework of accelerated bridge construction

- Optimization of performance-based maintenance plan.

Sustainability of repairs actions is a key issue to reduce the environmental impact during the whole lifecycles of deteriorating infrastructure. Toward this aim, Zirps et al. proposed a probabilistic framework aiming at ensuring optimal serviceability indicators for the design of civil infrastructure. The proposed approach combines, in an Excel-SIPmath ${ }^{\mathrm{TM}}$ environment, probabilistic service life models with lifecycles assessment to determine the times to repair and the environmental impact of 
repairs. The proposed approach is illustrated with a case study where it is highlighted the potential of using it for promoting sustainable and probabilistic-based design in engineering education and practice.

Climate change is modifying the environmental actions for structures and infrastructure systems. Therefore, lifetime assessment should consider these new environmental conditions that could modify the intensity and frequency of extreme loads and/or the kinetics of deterioration processes. Salman et al. studied the problem of maintenance of timber poles subjected to hurricanes and decay. They adopted a probabilistic approach for lifetime assessment and maintenance optimization that allows considering the different sources of uncertainty involved in the problem. The proposed approach was applied to two study cases in the United States: Miami, Florida, and New York City, New York. The results indicated that climate change could increase total maintenance costs. They also found that the consideration of preventive maintenance is crucial to decrease the failure rate.

Although climate changes and global warming bring an increase of average temperatures, huge amounts of de-icing salts are used on roads and bridges in continental climate due to stricter driving safety requirements during the winter season. Many chloride-ingress models have been developed in the last four decades, however, there are still plenty of opportunities to improve. Kušter Marić et al. present 3D chemo-hygro-thermomechanical (CHTM) model in which wetting-drying cycles, application of de-icing salts only in the winter season and cracks in concrete are considered in simulation of transport processes in a concrete structure. The model is applied on two motorway bridges in Croatia and numerical results match well with the chloride content measured on the bridges after 11 and 14 years of service. Modeling a realistic environmental condition results in the continuous transport processes in concrete and higher chloride content in deeper concrete layers as opposed to the models with the constant boundary conditions.

Cyclic loading is one of the main actions affecting the serviceability and safety of bridges worldwide. To deal with this problem, Stipanovic et al. proposed a probabilistic lifecycles model that takes into account structural safety, social and environmental impacts. The proposed approach was applied to a case study (steel bridge affected by fatigue) placed in the Netherlands. Four management strategies combining different actions (do minimum, strengthening, using temporal bridge, building a new bridge) were considered and compared in the example in terms of reliability, direct costs, user costs, and environmental impact. The results stress the potential of the proposed methodology to consider complex management strategies which is very useful for decision-making purposes.

Durability of a structure made of new materials or built by new construction methods is also an important issue presented in this Research Topic. As a modern material, ultra-highperformance concrete (UHPC) with excellent mechanical and durability properties, is suitable for new structures. Wang et al. studied the bending behavior of nine reinforced UHPC deck specimens exposed to four-point bending tests. Experiments show that the addition of steel fibers in UHPC improved the structure's toughness and crack resistance. Based on the test results, a theoretical formula is derived for calculating the bending stiffness of the UHPC decks during the entire loading process.

Accelerated bridge construction (ABC) is a building method using innovative design, materials and techniques to reduce construction time. Use of prefabricated bridge elements in the $\mathrm{ABC}$ results in many advantages, such as minimizing traffic disruptions and increasing safety for workers, vehicles, and the traveling public. However, closure joints connecting two adjacent prefabricated elements used to be vulnerable zone in terms of durability. Jaberi Jahromi et al. developed a framework for the service life design of closure joints in bridge decks, divided in seven steps and applied on a case study bridge. Several closure joints details are studied considering different concrete quality and rebars detailing. The mitigation strategies for service life design of closure joints are discussed and life cycle cost is analyzed.

Assessing the performance of structures in operation is still a challenge to ensure optimal levels of serviceability and safety during the whole lifetime. In situ tests with destructive, nondestructive or monitoring with embedded sensors are very useful toward this aim. Concerning structural health monitoring, Segura and Osma presented a mini-review about the potential use of micro-controllers for the development of solutions for the detection and quantification of corrosion processes in reinforced concrete structures. The authors state that these kinds of micro-controllers have been applied to other fields and that they could be adapted to develop low-cost and contact-less sensors for reinforced concrete structures. The information provided by these sensors is crucial to assess the structural condition under real exposure conditions as well as to verify/update service life models.

Collected papers in this research topic covered durability issues for different types of infrastructures (bridges, power distribution systems, etc.) made of different materials: concrete, steel and wood. Case studies from different sites of the world (United States, Norway, Netherlands, Croatia) are presented covering specific environment, climate and structure degradation mechanisms. Since development of numerical models for degradation is a complex process, provided laboratory experiments and selection of suitable measurement methods are also included in the research topic. Research presented here confirms the importance of service life prediction to achieve effective and efficient infrastructure management taking into account specific environmental conditions and climate changes.

\section{AUTHOR CONTRIBUTIONS}

All authors listed have made a substantial, direct, and intellectual contribution to the work and approved it for publication.

\section{ACKNOWLEDGMENTS}

Authors would like to thank all authors for their valuable contributions, the diligent reviewers and editors for their useful guidance to improve the papers. Special gratitude goes 
to the Editorial Team of Frontiers in Built Environment for the opportunity to launch this Research Topic and for the kind support through the whole project.

Conflict of Interest: The authors declare that the research was conducted in the absence of any commercial or financial relationships that could be construed as a potential conflict of interest.
Copyright $\odot 2021$ Kuster Marić and Bastidas-Arteaga. This is an open-access article distributed under the terms of the Creative Commons Attribution License (CC BY). The use, distribution or reproduction in other forums is permitted, provided the original author(s) and the copyright owner(s) are credited and that the original publication in this journal is cited, in accordance with accepted academic practice. No use, distribution or reproduction is permitted which does not comply with these terms. 